\title{
Mediastinal sarcoid lymphadenopathy in cancer patients: a diagnostic challenge
}

\begin{abstract}
Sarcoid-like reactions in lymph nodes have been described in cancer patients lacking systemic symptoms and signs of sarcoidosis. They are hypothesized to represent immune host response against the neoplastic cells. Their presence may hinder proper staging resulting in serious therapeutic ramifications, either in the form of overtreatment or less often under treatment. We present two cases of mediastinal lymphadenopathy in cancer patients that was presumed to be due to metastatic disease. Despite the administration of chemotherapy, the lesions persisted necessitating a biopsy that revealed their true nature. These clinical cases emphasize the importance of differential diagnosis of lymphadenopathy in the context of malignant disease.
\end{abstract}

Keywords: sarcoidosis, lymphadenopathy, mediastinum
Volume 2 Issue 3 - 2015

\author{
Kamperis E,' Asteriou C, ${ }^{2}$ Kleontas A, ${ }^{2}$ \\ Barbetakis $\mathrm{N}^{2}$ \\ 'Radiation Oncology Department Theagenio Cancer Hospital, \\ Greece \\ ${ }^{2}$ Thoracic Surgery Department, Theagenio Cancer Hospital, \\ Greece
}

Correspondence: Efstathios Kamperis, Radiation Oncology Department Theagenio Cancer Hospital, Al Simeonidi 2, PO 55535, Thessaloniki, Greece, Tel +30 2310 898313, Fax +30 2310 898655, Email ekamperi@gmail.com

Received: October 18, 2014 | Published: March 23, 2015
Abbreviations: VATS, video-assisted thoracoscopic surgery; EUS-FNA, endoscopic ultra-sound fine-needle aspiration; EBUSTBNA, endobronchial ultra-sound transbronchial needle aspiration

\section{Introduction}

The presence of non-caseating granulomas in cancer patients and no symptoms or signs suggestive of systemic sarcoidosis is typically referred to as sarcoid-like reaction. Such reactions are relatively often in patients with underlying malignancies and can be found either in the site of primary tumor or in the regional lymph nodes. They are thought to represent an immune defense mechanism against tumor cells. Their significance lies in how they can masquerade as metastatic disease leading to overtreatment. Here we describe two cancer patients with persistent sarcoid-like mediastinal lymphadenopathy that mimicked disease progression and shortly review the relevant literature.

\section{Case report}

\section{Case I}

A 45-year old woman presented with a left palpable breast mass. Her medical record included hormone replacement therapy for nine years and an appendectomy. Mammography was suggestive of a malignant process and percutaneous biopsy with Temno ${ }^{\circledR}$ needle revealed a low-grade invasive ductal carcinoma not otherwise specified (NOS). On immunostaining the tumor was estrogen and progesterone negative and HER2/neu positive. The rest of the staging was unremarkable, except for the presence of marginally enlarged mediastinal lymph nodes on CT chest scan.

The patient received three cycles of neoadjuvant chemotherapy (paclitaxel $80 \mathrm{mg} / \mathrm{m}^{2}$ day $1,8,15$ ) concurrently with trastuzumab $4 \mathrm{mg} / \mathrm{kg}$ loading dose intravenously, then $2 \mathrm{mg} / \mathrm{kg}$ weekly. Upon reevaluation, the clinical and mammographic findings were congruent with a complete response. A ${ }^{99} \mathrm{mTc}$-MIBI breast scintigraphy ${ }^{1}$ was also negative compared to the baseline that had been obtained prior to the commencement of chemotherapy. A repeat CT scan of the chest however did not show any recession of the lymph nodes. The patient therefore underwent a video-assisted thoracoscopic surgery (VATS) and a mini thoracotomy. Intraoperatively, a nodule in the right upper lobe was detected and removed. The frozen section biopsy was negative for malignancy. Subsequently, the upper paratracheal, lower paratracheal and subcarinal lymph nodes were sampled. The operation was uneventful and the patient was discharged the $9^{\text {th }}$ post-operative day. Microscopic examination of both the resected lung nodule and the lymph nodes showed multiple non-caseating granulomas, along with giant cells of Langhans and foreign-body types. There were no evidence of malignancy.

Since metastatic disease had been ruled out, our patient underwent segmental mastectomy with axillary lymph node dissection. The histological assessment failed to reveal any residual disease either in the breast or in the axillary lymph nodes specimens. She completed the adjuvant treatment with paclitaxel/trastuzumab and received radiotherapy with two tangential breast fields at a total dose of 50.4Gy with $1.8 \mathrm{~Gy}$ dose per fraction. Approximately four years after the initial diagnosis, the patient exhibits no evidence of relapsed breast cancer or systemic manifestations of sarcoidosis, despite the persistent but stable CT-documented mediastinal lymphadenopathy.

\section{Case 2}

A 68-year old woman was admitted to our hospital after having been diagnosed with a grade II bilateral endometrioid ovarian cancer metastatic to omentum and Douglas pouch (stage IIIC). She had undergone total abdominal hysterectomy with bilateral salpingooophorectomy in another hospital facility. Her medical record included cholecystectomy, appendectomy and rheumatic mitral stenosis. Her mother had non-Hodgkin's lymphoma.

After receiving six cycles of adjuvant chemotherapy (paclitaxel $175 \mathrm{mg} / \mathrm{m}^{2}$, carboplatin AUC5), a CT scan of the chest revealed generalized mediastinal and hilar lymphadenopathy with punctate calcifications in some of the lymph nodes and enlarged intrapulmonary lymph nodes. Her CA-125 level was $18.29 \mathrm{U} / \mathrm{ml}$ (pre-treatment level $40.02 \mathrm{U} / \mathrm{ml}$ ). Considering the fact that the rest of her clinical and radiological evidence were not consistent with a disease progression, she underwent a VATS with sampling of a subcarinal lymph node block. The operation was uneventful and the patient was discharged the $5^{\text {th }}$ post-operative day. 
Microscopic examination of the resected lymph nodes revealed numerous dense non-caseating granulomas of variable size and shape. They consisted of epithelioid cells and rarely multinuclear giant cells. There were no cytoplasmic inclusions, such as asteroid bodies or Schaumann corpuscles, nor any signs of neoplastic disease. The patient continues her adjuvant chemotherapy treatment, without any overt symptoms or signs of sarcoidosis.

\section{Discussion}

Sarcoidosis is a multi-organ inflammatory disease of unknown cause. It is largely a diagnosis of exclusion, suggested by the presence of certain clinical symptoms, radiological findings and histological evidence of non-caseating epithelioid-cell granulomas. Although virtually any organ may be affected, lung, skin and eyes are more frequently involved. Symptoms include dyspnea, cough, wheezing, macules, papules and plaques, erythema nodosum and uveitis. Chest imaging findings range from bilateral symmetric hilar lymphadenopathy and reticular opacities to lung fibrosis and honeycombing.

Other features suggestive of sarcoidosis are increased angiotensin converting enzyme concentration, a raised $\mathrm{CD} 4 / \mathrm{CD} 8$ ratio in bronchoalveolar lavage, panda sign on gallium scan, lupus pernio and Löfgren's syndrome. The etiopathogenesis of sarcoidosis remains unknown, with the three most prevalent theories being genetic predisposition, immune dysregulation and environmental exposure. ${ }^{2}$ Non-caseating tumor-related granulomas may occasionally develop in individuals lacking any symptoms or signs of systemic sarcoidosis. Such histological features are designated as "sarcoid-like" reactions in the context of malignant disease. ${ }^{3}$ They are found along the lymph node drainage path, to the stroma of a primary solid tumor and less often to distant lymph nodes, bone marrow, spleen and liver, ${ }^{4}$ usually distributed in a paravascular location.

The incidence of sarcoid-like reactions was estimated by Brincker $\mathrm{H}^{4}$ after reviewing data of 4,020 patients with diverse malignant neoplasms and found that $4.4 \%$ (165 of 3,770) patients with carcinoma had such lesions. As far as the histological type is concerned, squamous cell carcinomas were marginally more likely to be associated with a sarcoid-like reaction over adenocarcinomas, while sarcomas showed the least correlation, with only one case out of a total of 250. Interestingly, sarcoidal reactions were four times more likely to occur in metastases-free lymph nodes, than in lymph nodes with metastatic disease. Likewise, Steinfort DP and Irving LB ${ }^{6}$ found that they occurred solely in patients with stage I disease in their non-small cell lung cancer case series, which might imply that they constitute an early event in the course of malignancy.

Granulomas consist of a cluster of macrophages formed in an attempt of the immune system to confine entities that it perceives as alien but is unable to eliminate. Sarcoid reactions are likely a T-cell mediated response ${ }^{7}$ of the immune system to soluble antigenic factors released from tumor cells, ${ }^{4}$ whereas others regard it as a local degenerative process incited by the necrosis of the tumor itself. ${ }^{8}$ There have been cases of therapy-induced sarcoid-like reactions, associated with a wide variety of cytotoxic chemotherapeutics (cisplatin, bleomycin, paclitaxel), immunomodulating agents (alpha-interferon, interleukin-2) and radiotherapy. ${ }^{9,10}$ The reason why antineoplastic treatment appears to evoke such reactions or amplify existing ones is probably due to increased tissue breakdown or direct modulation of the immune system.

Distinguishing a tumor-related sarcoid-like reaction from sarcoidosis is based on the lack of systemic symptoms but it is not always entirely trivial. Anorexia, weight loss, fever, cough, dyspnea or other non-specific constitutional symptoms are common in cancer patients and may overlap with those of sarcoidosis. It has been suggested that the distinction could be made at a microscopic level. In specific, the presence of B-cells could divide the space of granulomas into B-cell positive (sarcoid-like reactions, toxoplasmosis) and B-cell negative (sarcoidosis, mycobacterial infection). ${ }^{4} \mathrm{~A}$ more recent study however invalidated the above bisection suggesting that the histological appearance and cell distribution of sarcoid-like reactions are similar to other granulomatous diseases, including sarcoidosis. ${ }^{7}$ There is also vast spectrum of lung pathology that manifests itself as a granulomatous condition, most notably fungal infections, mycobacterial infections, sarcoidosis, rheumatoid arthritis, Wegener's disease and neoplasia that needs to be taken into account, when considering the differential diagnosis.

Identifying sarcoid-like reactions as metastasis results in disease upstaging. This in turn could lead to overtreatment risking treatmentrelated toxicities and death ${ }^{11}$ or to under treatment due to the referral of the patient to conservative/palliative therapy, when a more aggressive course of treatment should be pursued. Contrariwise, it is possible for a sarcoid-like reaction to encompass micrometastatic disease that can be missed and treatment be withheld. Therefore, adequate material from multiple biopsy sites, a comprehensive pathologic examination and immunohistological analysis are all necessary to avoid potential pitfalls. $^{12}$

There are various diagnostic modalities for staging mediastinal lymph nodes, including mediastinoscopy ${ }^{13,14}$ video-assisted thoracoscopic surgery (VATS), ${ }^{14-16}$ transesophageal endoscopic ultrasound fine-needle aspiration (EUS-FNA) ${ }^{17}$ and endobronchial ultrasound transbronchial needle aspiration (EBUS-TBNA). ${ }^{6,18}$ Each method has its own set of advantages and disadvantages, depending on the clinical context (patient comorbidities, lymph node stations of interest, availability, experience, etc).

Aide suggested metabolic response of sarcoid-like lesions on FDG-PET to short corticosteroid therapy as a means to differentiate them from metastatic disease. ${ }^{19}$ In specific, oral administration of prednisolone 1-2 mg/kg/day over 14-16 days was enough to induce a complete disappearance of FDG uptake foci in their two studied cases. In order for this method to produce valid results 1 . Other infectious diseases need to be ruled out (primarily tuberculosis) and 2. Tumor should not be a lymphoma, where the decrease of FDG uptake in both inflammatory and cancer tissue is expected after corticosteroid treatment.

The role of sarcoid-like reactions in cancer prognosis is not consistent across all tumor types. In Hodgkin's disease patients there is a trend towards fewer relapses and longer survival..$^{5,20}$ In contrast, lung cancer patients ${ }^{21}$ and breast cancer patients ${ }^{22}$ do not appear to benefit much. There is a remarkable case though reported by Lynch of a patient with small-cell lung cancer that presented itself as pulmonary granulomas, remaining undetected and therefore untreated for twelve months despite the meticulous diagnostic work-up. ${ }^{23}$ The authors suggest that maybe the sarcoidal reactions represented a host-defense mechanism against the neoplasm.

\section{Conclusion}

In conclusion, sarcoid-like reactions must be considered in the differential diagnosis of patients with a history of malignancy that develops hypermetabolic lymphadenopathy, particularly 
when the latter is resilient to antineoplastic treatment. Thorough histopathological examination can be useful for establishing a correct diagnosis and determining the most appropriate line of treatment.

\section{Acknowledgments}

None.

\section{Conflicts of interest}

Authors declare that there is no conflict of interest.

\section{References}

1. Maini CL, Tofani A, Sciuto R, et al. Technetium-99m-MIBI scintigraphy in the assessment of neoadjuvant chemotherapy in breast carcinoma. $J$ Nucl Med. 1997;38(10):1546-1551.

2. Baughman RP, Lower EE, du Bois RM. Sarcoidosis. Lancet. 2003;361(9363):1111-1118.

3. Symmers WS. Localized tuberculoid granulomas associated with carcinoma. Am J Pathol. 1950;27(3):493-521.

4. Brincker H. Sarcoid reactions in malignant tumours. Cancer Treatment Rev. 1986;13(3):147-156.

5. Sacks EL, Donaldson SS, Gordon J, et al. Epithelioid granulomas associated with Hodgkin's disease. Cancer. 1978;41(2):562-567.

6. Steinfort DP, Irving LB. Sarcoidal reactions in regional lymph nodes of patients with non-small cell lung cancer: incidence and implications for minimally invasive staging with endobronchial ultrasound. Lung Cancer. 2009;66(3):305-308.

7. Kurata A, Terado Y, Schulz A, et al. Inflammatory cells in the formation of tumor-related sarcoid reactions. Hum Pathol. 2005;36(5):546-554.

8. Llombart AJ, Escudero MJ. The incidence and significance of epithelioid and sarcoid-like cellular reaction in the stromata of malignant tumours. A morphological and experimental study. Eur J Cancer. 1970;6(6):545-551.

9. Trump DL, Ettinger DS, Feldman MJ, et al. "Sarcoidosis" and Sarcoidlike Lesions. Their Occurrence After Cytotoxic and Radiation Therapy of Testis Cancer. Arch Intern Med. 1981;141(1):37-38.

10. Butt S, Alzebdeh R, Kable TD, et al. Non-caseating granulomas in patients after the diagnosis of cancer: clinical characteristics and outcome. Sarcoidosis Vasc Diffuse Lung Dis. 2011;28(1):44-49.
11. Kok TC, Haasjes JG, Splinter TA, et al. Sarcoid-like lymphadenopathy mimicking metastatic testicular cancer. Cancer. 1991;68(8):1845-1847.

12. Tjan-Heijnen VCG, Vlasveld LT, Pernet FPPM, et al. Clinical case Coincidence of seminoma and sarcoidosis: A myth or fact? Ann Oncol. 1998;9(3):321-325.

13. Hunt BM, Vallières E, Buduhan G, et al. Sarcoidosis as a benign cause of lymphadenopathy in cancer patients. Am J Surg. 2009;197(5):629-632.

14. Rendina EA, Venuta F, Giacomo T, et al. Comparative merits of thoracoscopy, mediastinoscopy, and mediastinotomy for mediastinal biopsy. Ann Thorac Surg. 1994;57(4):992-995.

15. Landreneau RJ, Mack MJ, Dowling RD, et al. The Role of Thoracoscopy in Lung Cancer Management. Chest. 1998;113(1 Suppl):6S-12S

16. Massone PP, Lequaglie C, Magnani B, et al. The Real Impact and Usefulness of Video-Assisted Thoracoscopic Surgery in the Diagnosis and Therapy of Clinical Lymphadenopathies of the Mediastinum. Ann Surg Oncol. 2003;10(10):1197-1202.

17. Eloubeidi MA, Cerfolio RJ, Chen VK, et al. Endoscopic ultrasoundguided fine needle aspiration of mediastinal lymph node in patients with suspected lung cancer after positron emission tomography and computed tomography scans. Ann Thorac Surg. 2005;79(1):263-268.

18. Kennedy MP, Jimenez CA, Mhatre AD, et al. Clinical implications of granulomatous inflammation detected by endobronchial ultrasound transbronchial needle aspiration in patients with suspected cancer recurrence in the mediastinum. J Cardiothoracic Surg. 2008;3:8.

19. Aide N, Allouache D, Ollivier Y, et al. Early 2'-deoxy-2'-[18F] fluoroD-glucose PET metabolic response after corticosteroid therapy to differentiate cancer from sarcoidosis and sarcoid-like lesions. Mol Imaging Biol. 2009;11(4):224-228.

20. O’Connell MJ, Schimpff SC, Kirschner RH, et al. Epithelioid Granulomas in Hodgkin Disease. JAMA. 1975;233(8):886-889.

21. Tomimaru Y, Higashiyama M, Okami J, et al. Surgical results of lung cancer with sarcoid reaction in regional lymph nodes. Jpn J Clin Oncol. 2007;37(2):90-95.

22. Martella S, Lohsiriwat V, Barbalho DM, et al. Sarcoid-like reaction in breast cancer: a long-term follow-up series of eight patients. Surg Today. 2012;42(3):259-263.

23. Lynch JP, Flint A, Miller DM, et al. Noncaseating pulmonary granulomas associated with small cell carcinoma of the lung. Am J Med. 1985;78(4):691-696. 